\title{
L'index de sélection sur plusieurs caractères : une synthèse sur les pondérations économiques, le choix de contraintes et de caractères associés
}

\author{
J.-P. Sampoux \\ CNRS-INRA-UPS, ferme du Moulon, 91190 Gif-sur-Yvette, France
}

(reçu le 9 février 1989, accepté le 31 août 1989)

\begin{abstract}
Résumé - L'index de sélection sur plusieurs caractères est classiquement le meilleur prédicteur linéaire d'une combinaison linéaire de valeurs génétiques des caractères considérés. Les coefficients de cette combinaison linéaire ont d'abord été définis comme étant les valeurs économiques unitaires relatives de ces caractères. Mais la définition d'une valeur économique globale est parfois difficile ou inadaptée. Il est alors préférable de chercher un système de contraintes portant sur les espérances de progrès génétiques pour certains caractères. De telles contraintes peuvent également être combinées ou non à l'amélioration d'une valeur économique globale. La recherche du système de contraintes le moins restrictif possible est nécessaire pour obtenir les progrès génétiques les plus élevés compatibles avec l'objectif de sélection. Pour cela, il est possible de ne définir, pour certains caractères, qu'une limite inférieure ou supérieure à l'espérance de progrès génétique. Ce dernier type de contraintes trouve un intérêt tout particulier pour contrôler la réponse à la sélection de caractères associés, observés dans le but d'augmenter l'efficacité de la sélection sur d'autres caractères. Lorsqu'un progrès génétique est recherché sur plusieurs caractères, il est d'autre part montré que la précision de l'estimation de leur valeur génétique n'est pas un critère suffisant pour juger de l'intérêt de caractères associés.
\end{abstract}

index de sélection - pondérations économiques - gains désirés relatifs - contraintes d'inégalité - caractères associés

Summary - The selection index for several traits : an assessment of economic weights, and the choice of constraints and associated traits. The selection index for several traits is generally the best linear predictor of a linear combination of genetic values of traits taken into account. The coefficients of this linear combination were first defined as relative economic values of these traits. While defining overall economic value is often hard or unsuitable, it is easier to look for a constraint system based upon expected genetic progress for certain traits. Such a constraint system can either be combined or not with the improvement of an overall economic value. It is therefore necessary to look for the least restrictive constraint system in order to achieve the best genetic gains compatible with the selection aim (Fig. 1). For this purpose, only inferior or superior boundaries of expected gains need be given for some traits. The latter type of constraint is especially useful for controlling selection responses of associated traits, which have been observed to increase selection efficiency for other traits (Table I). When genetic gains are required for several traits, it has been shown that the efficiency of associated traits cannot be checked on the single basis of the accuracy of the estimation of their genetic value $\left[r^{2}(G, G)\right]$, but expected gains with the chosen constraint system should also be taken into account (Table I/ \& Fig. 2). However, an estimation of the expected accuracy of these values is required in order to determine the efficiency of associated traits.

selection index - economic weights - desired relative gains - inequality constraints - associated traits

\section{INTRODUCTION}

Dans un programme de sélection récurrente, plusieurs caractères d'intérêt économique doivent en général être pris en considération. Lorsqu'il s'agit d'améliorer une valeur économique globale s'exprimant comme une combinaison linéaire des valeurs génétiques de différents caractères, la sélection sur index est au moins aussi efficace que la sélection par niveaux indépendants, ellemême toujours plus efficace que la sélection tandem (Gallais, 1973). D'une façon générale, l'optimisation de la sélection par niveaux indépendants est difficile quand le nombre de 
caractères à améliorer est grand. Une sélection sur la seule valeur d'un index, combinaison linéaire des valeurs observées de différents caractères, est alors d'une réalisation plus simple, pour autant que les coefficients de cette combinaison linéaire puissent être déterminés de façon pertinente.

La détermination des pondérations de l'index dépend d'une part d'une estimation suffisamment précise de la valeur génétique des caractères à améliorer (Vincourt \& Gallais, 1983), et d'autre part de l'attribution d'une valeur économique relative à chacun de ces caractères (Rouvier, 1977). Ces valeurs économiques relatives peuvent être déterminées au préalable en fonction de l'importance des différents caractères considérés dans le contexte économique et technique visé. La définition d'un système de contraintes appliquées aux espérances de progrès génétiques des caractères à améliorer peut également contribuer à la pondération de ces caractères. Les principaux systèmes de contraintes employés sont exposés et leur intérêt est discuté. Quelques conséquences et perspectives d'application concernant l'emploi de caractères associés sont envisagées à partir d'exemples de sélection en amélioration des plantes.

\section{Hypothèses de l'étude}

Chaque individu candidat à la sélection est caractérisé par le vecteur [G] des valeurs génétiques additives non connues des caractères à améliorer ainsi que par un vecteur $[P]$ des valeurs phénotypiques centrées de caractères observés sur l'individu lui-même ou sur des apparentés. II est supposé une distribution multinormale du vecteur de variables aléatoires [G/P], ou du moins la linéarité des liaisons entre variables. Les variances et covariances génétiques et phénotypiques, ainsi que les covariances entre valeurs génétiques et variables phénotypiques observées sont supposées connues exactement.

\section{DÉTERMINATION DES PONDÉRATIONS DE L'INDEX DE SÉLECTION POUR DIFFÉRENTS SYSTÈMES DE CONTRAINTES}

\section{Index de sélection pour l'amélioration d'une valeur économique globale}

Initialement, la sélection sur index fut conçue pour améliorer une valeur économique globale (H). Celle-ci était définie comme une combinaison linéaire des valeurs génétiques pour les dif- férents caractères d'intérêt économique (Smith, 1936; Hazel, 1943). Pour un individu $j$ de la population:

$$
\mathrm{H}_{j}=\sum_{i=1, g} a_{i} \mathrm{G}_{i j}
$$

$\mathrm{G}_{i j}$ est la valeur génétique de l'individu $j$ pour le caractère $i$. Les coefficients $a_{i}$ peuvent être définis comme les coefficients de régression linéaire multiple de la valeur économique globale $\mathrm{H}$ sur les valeurs génétiques $G_{i}$.

Pratiquement, la valeur génétique est estimée par régression linéaire multiple sur les valeurs phénotypiques centrées observées $\left(P_{1}, \ldots P_{p}\right)$.

$$
\left[\begin{array}{c}
\widehat{G}_{1} \\
\vdots \\
\widehat{G}_{g}
\end{array}\right]=\Sigma_{G P} \Sigma_{P P}^{-1}\left[\begin{array}{c}
P_{1} \\
: \\
P_{P}
\end{array}\right]
$$

$\Sigma_{\mathrm{GP}}$ est la matrice de variance - covariance entre valeurs génétiques à prédire et valeurs phénotypiques prédictrices.

$\Sigma_{\mathrm{PP}}$ est la matrice de variance - covariance entre variables phénotypiques prédictrices.

L'index de sélection utilisé est donc naturellement :

$$
\mathrm{I}=\sum_{i=1, g} a_{i} \widehat{\mathrm{G}}_{i}
$$

Cet index est également une combinaison linéaire des valeurs phénotypiques observées:

$$
\mathrm{I}=\sum_{I=1, p} b_{l} P_{l}
$$

Les coefficients $b_{l}$ se déduisant des coefficients $a_{i}$ par l'application :

$$
\left[b_{l}\right]=\left(\Sigma_{G P} \Sigma_{P P}^{-1}\right)\left[a_{i}\right]
$$

Cette méthode d'établissement de l'index de sélection sur plusieurs caractères et donc d'estimation de la valeur économique globale a été établie par Rouvier (1969). Cet auteur considérait que les valeurs phénotypiques moyennes étaient exactement connues. Lorsque les valeurs moyennes phénotypiques sont inconnues et dans le cas d'informations inégales (déséquilibre des effectifs de famille), Henderson (1963, 1975) a introduit en sélection animale les notions de meilleur prédicteur linéaire sans biais (BLUP) de la valeur génétique et d'estimation des effets fixés pour les différents caractères à améliorer.

En amélioration des plantes, de tels index ont été relativement peu utilisés. La valeur économique relative d'un caractère (coefficient $a_{i}$ ) peut en effet être difficile à percevoir à travers des considérations qui ne sont pas nécessairement 
objectives. Elle peut de plus évoluer, avec les années, ou à l'intérieur de la gamme de variation génétique étudiée.

\section{Index de sélection pour l'obtention de gains désirés relatifs}

\section{Le problème à résoudre}

Pour le sélectionneur, il est en fait souvent préférable de se fixer un objectif en termes de progrès génétique à atteindre sur chaque caractère d'intérêt économique. Un tel type de contraintes peut en effet permettre de fixer les pondérations relatives des caractères à améliorer (coefficients $a_{i}$ ). Différents types de contraintes sont envisageables. Le problème est de déterminer les pondérations $a_{i}$ conduisant à l'index des valeurs phénotypiques $I=\Sigma_{l} b_{1} P$, qui procure les progrès génétiques attendus les plus élevés, tout en satisfaisant au système de contraintes imposé.

\section{Expression de la valeur des progrès géné- tiques attendus}

Les progrès génétiques attendus $\Delta G$ peuvent être exprimés :

- en fonction des coefficients $b_{1}$ appliqués aux valeurs phénotypiques observées :

$$
\Delta \mathrm{G}=i / \sigma_{l} \operatorname{cov}(\mathrm{G}, l)=i / \sigma_{l} \Sigma_{\mathrm{GP}}\left[b_{l}\right]
$$

où $\sigma_{1}$ est l'écart type de l'index $\left(\sigma_{1}^{2}=b^{\prime} \Sigma_{\mathrm{pp}} b\right)$, et $i$ est l'intensité de sélection en unité d'écart-type.

- en fonction des coefficients $a_{i}$ appliqués aux valeurs génétiques des caractères soumis à contrainte, estimées par la régression sur les valeurs phénotypiques observées :

$$
\Delta \mathrm{G}=i / \sigma_{\mathrm{l}} \Sigma_{\hat{\mathrm{G}} \hat{\mathrm{G}}}\left[a_{i}\right]
$$

$\Sigma_{\widehat{G}}=$ matrice de variance - covariance entre valeurs génétiques vraies et valeurs estimées.

$$
\Sigma_{\widehat{G}}=\Sigma_{\widehat{G} \hat{G}}=\Sigma_{G P} \Sigma_{P P}^{-1} \Sigma_{P G} \text { (Rouvier, 1969) }
$$

\section{L'ellipsoïde des espérances de progrès géné- tiques}

Soit $g$ le nombre de caractères soumis à contrainte. Dans l'espace affine de dimension $g$, chaque index $I=\Sigma_{i=1, g} a_{i} \widehat{G}_{i}$ peut être caractérisé par un point dont les coordonnées $[\Delta G]$ sont égales aux progrès génétiques attendus sur les caractères soumis à contrainte. Lorsque les coefficients $a_{i}$ prennent l'ensemble des valeurs relatives possibles, le point $[\Delta G]$ décrit un ellipsoïde d'équation :

$$
[\Delta \mathrm{G}] \cdot \Sigma_{\hat{\mathrm{G}} \hat{\mathrm{G}}}^{-1}[\Delta \mathrm{G}]=i^{2}
$$

\section{Les contraintes de stricte proportionnalité}

Un premier type de contraintes peut être que les progrès génétiques attendus pour certains caractères $(\Delta \mathrm{G})$ soient proportionnels à des valeurs $k$ choisies par le sélectionneur :

$$
\left[\Delta \mathrm{G}_{i}\right]=\lambda\left[k_{i}\right]
$$

Lorsque la dimension du vecteur $\left[k_{i}\right]$ est égale à celle du vecteur $\left[P_{l}\right]$, la détermination des coefficients $b_{\text {l }}$ de l'index satisfaisant au système de contraintes (3) est immédiate. Le système :

$$
\Sigma_{\mathrm{GP}}\left[b_{1}\right]=\left[k_{i}\right]
$$

est en effet un système dont le nombre d'inconnues (coefficients $b_{l}$ ) est égal au nombre d'équations. Si la matrice $\Sigma_{\mathrm{GP}}$ est inversible, un vecteur $[b]$ unique est solution du système. Cette méthode de détermination des coefficients de l'index a été proposée par Pesek \& Baker (1969). Dans ce cas, l'application (1) est également bijective. L'in$\operatorname{dex} \Sigma_{l} b_{l} P_{l}$ correspondant au vecteur [b] solution du système (4) est donc toujours une combinaison linéaire des valeurs génétiques estimées par régression sur les valeurs phénotypiques observées $\left(\Sigma_{i} a_{i} \widehat{G}_{i}\right)$.

Lorsque la dimension du vecteur $\left[P_{l}\right]$ est supérieure à celle du vecteur $\left[k_{i}\right]$, le système (4) n'a pas de solution unique. Les coefficients de l'index le plus efficace sont obtenus par la résolution du système :

$$
\Sigma_{\widehat{Q} \hat{G}}\left[a_{i}\right]=\left[k_{i}\right] \text { (Rouvier, 1977) }
$$

le vecteur [b] se déduisant du vecteur [a] par l'application (1).

Ces contraintes de stricte proportionnalité sont en fait assez difficiles à utiliser. Elles ne conduisent pas à la sélection la plus efficace lorsque l'on souhaite simplement, pour certains caractères, que le niveau moyen de la population se situe au-delà d'un seuil critique, celui-ci pouvant être défini en valeur relative par rapport à d'autres caractères.

Ceci peut être vérifié par l'exemple simple de la sélection sur deux caractères (Fig. 1). Pour le caractère 2 , nous supposerons que l'on souhaite uniquement éviter une diminution de la valeur moyenne de la population. Une contrainte du type

$$
\left[\begin{array}{l}
\Delta \mathrm{G}_{1} \\
\Delta \mathrm{G}_{2}
\end{array}\right]=\lambda\left[\begin{array}{l}
k_{1} \\
k_{2}
\end{array}\right]
$$

avec $k_{1}$ et $k_{2}$ positifs, permet d'éviter une telle dérive. Choisie a priori, elle ne conduit pas 

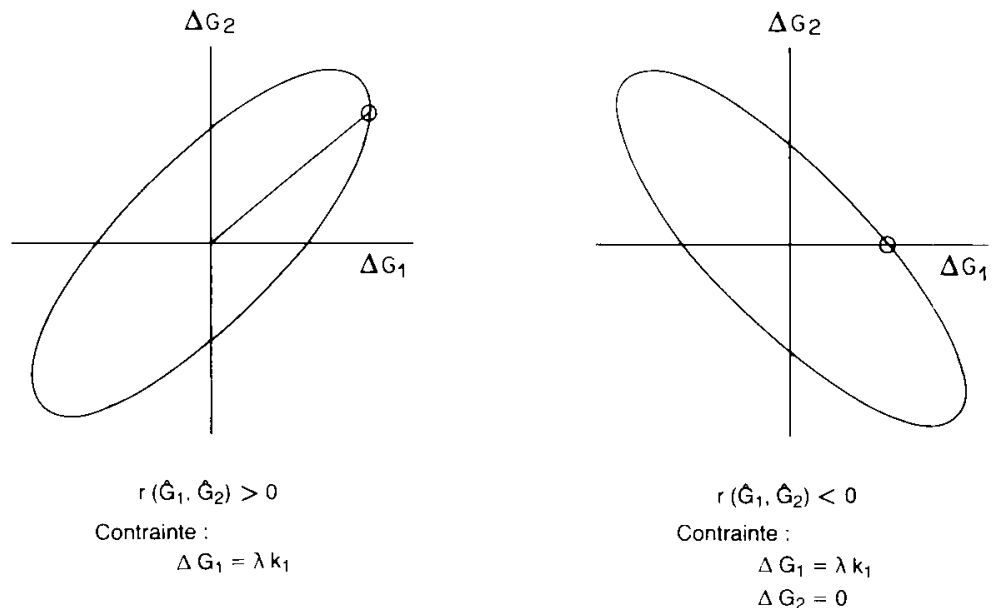

Fig. 1. Système optimal de contraintes de stricte proportionnalité pour un objectif de sélection visant à maximiser le progrès génétique attendu sur le caractère 1, en évitant une dégradation de la valeur moyenne pour le caractère 2.

nécessairement à l'efficacité maximale (c'est-àdire obtenir le progrès génétique maximal sur le caractère 1 , compte tenu de la contrainte $\Delta \mathrm{G}_{2} \geq 0$ ). La connaissance de la matrice de variance - covariance entre prédicteurs $\left(\Sigma_{\hat{G} \hat{G}}\right)$, donc de l'ellipse d'égale densité de probabilité des espérances de progrès génétiques, est nécessaire pour déterminer les valeurs optimales de $k_{1}$ et de $k_{2}$. Cette détermination n'est toutefois évidente que dans le cas de deux caractères soumis à contraintes.

\section{Les contraintes d'inégalités relatives}

Des limites inférieures ou supérieures aux espérances de progrès génétiques peuvent être formalisées par des contraintes d'inégalités relatives :

$$
\left\{\begin{aligned}
\Delta G_{1} & =\lambda k_{1} \\
\Delta G_{2} & \geq \lambda k_{2} \\
\Delta G_{3} & \leq \lambda k_{3} \\
\lambda k_{4} \leq \Delta G_{4} & \leq \lambda k_{5}
\end{aligned}\right.
$$

(Chaque ligne peut être une égalité ou une inégalité vectorielle, c'est-à-dire représenter un groupe de contraintes imposées sur différents caractères).

II existe évidemment une infinité de systèmes de contraintes de stricte proportionnalité vérifiant un système donné de type 5 . Le meilleur index est celui qui est le plus efficace pour le système de contraintes de stricte proportionnalité optimal.

Une méthode de détermination des coefficients $b_{1}$ de l'index $\Sigma_{1} b_{1} P_{1}$ le plus efficace pour un système de contraintes du type 5 a été proposée par Itoh \& Yamada (1988). L'expression de la valeur des progrès génétiques attendus montre que $\lambda$ est inversement proportionnel à l'écart type de l'index. C'est donc l'index de variance $\left(\sigma_{1}^{2}=b^{\prime} \Sigma_{\mathrm{Pp}} b\right)$ minimal qui permet d'atteindre les progrès génétiques les plus élevés. Les coefficients de l'index le plus efficace peuvent alors être déterminés à l'aide d'un algorithme de programmation quadratique. Cet index est toujours une combinaison linéaire des valeurs génétiques des caractères soumis à contrainte, estimées par régression sur les valeurs phénotypiques observées. Pour obtenir les progrès génétiques les plus satisfaisants, le sélectionneur peut donc simplement rechercher le système de contraintes du type 5 le moins restrictif possible pour son objectif de sélection, l'index le plus efficace étant ensuite déterminé par la méthode de Itoh \& Yamada.

\section{Index de sélection sur une valeur écono- mique globale avec restrictions}

Un compromis entre une sélection sur une valeur économique globale et une sélection pour des gains désirés peut être réalisé en ajoutant au système (5) une contrainte de progrès sur une valeur économique globale $\left(\Delta G_{H}\right)$ :

$$
\left\{\begin{aligned}
\Delta \mathrm{G}_{1} & =\lambda k_{1} \\
\Delta \mathrm{G}_{2} & \geq \lambda k_{2} \\
\Delta \mathrm{G}_{3} & \leq \lambda k_{3} \\
\lambda \mathrm{k}_{4} \leq \Delta \mathrm{G}_{4} & \leq \lambda \mathrm{k}_{5} \\
\Delta \mathrm{G}_{\mathrm{H}} & =\lambda k_{6} \quad \text { (ou } \Delta \mathrm{G}_{H} \text { maximum) }
\end{aligned}\right.
$$

Une méthode de détermination du meilleur index satisfaisant à un tel système a été proposée par Mallard (1972), lorsque la valeur économique globale est une combinaison linéaire des valeurs génétiques de plusieurs caractères. Comme dans le cas précédent, elle conduit à la minimisation d'une forme quadra- 
tique. Les contraintes d'inégalités présentent le même avantage que dans le cas d'une sélection dont l'objectif est uniquement défini en termes de gains désirés.

Des systèmes de contrainte du type 6 ont été imaginés de façon à sélectionner avec davantage de sécurité lorsque la valeur économique globale est difficile à définir avec précision, ou encore pour les cas où l'on souhaite imposer une contrainte sur un caractère ne participant pas à la définition de la valeur économique globale. Toutefois, l'emploi de restrictions (contraintes autres que la maximisation de $\Delta G_{H}$ ) peut limiter considérablement le progrès génétique sur la valeur économique globale. Dans certains cas, il peut être préférable d'abandonner la contrainte de progrès génétique sur une valeur économique globale mal définie, et de se limiter à rechercher un bon ajustement des progrès génétiques attendus sur les différents caractères.

\section{CORRECTION DES COEFFICIENTS DE L'IN- DEX LORSQUE DES CARACTERÈS ASSO- CIÉS SONT OBSERVÉS}

Différents caractères associés, liés aux caractères à améliorer, peuvent être observés de façon à améliorer l'efficacité de la sélection. Lorsque l'amélioration de la population n'est recherchée que pour un caractère, les coefficients de l'index appliqués aux valeurs phénotypiques peuvent alors simplement être les coefficients de la régression génotype sur phénotype. Toutefois, la prise en compte des valeurs phénotypiques des caractères associés entraîne une augmentation de leur réponse corrélée à la sélection. La valeur moyenne optimale de la population pour ces caractères peut alors être atteinte avant celle des caractères principaux. Un seuil critique peut même être dépassé. La prise en compte d'un système de contraintes de type 5 peut permettre de modifier les coefficients de l'index de façon à éviter une telle dégradation de la valeur de la population pour les caractères associés.

Ceci peut être illustré par un exemple tiré d'une expérimentation maïs fourrage réalisée au GS Moulon. La valeur en croisement des individus d'une population de maïs précoce est appréciée par une récolte en plante entière de leurs descendances en croisement avec un testeur. La sélection est réalisée sur un index des valeurs génétiques estimées du rendement et de la teneur en matière sèche du produit récolté. Les coefficients de l'index sont déterminés de façon à maintenir le niveau moyen de précocité (apprécié à travers la teneur en matière sèche). L'observa- tion de caractères associés sur les descendances en croisement (hauteur totale des plantes, hauteur de l'épi, longueur de la feuille de l'épi et date de floraison femelle) permet d'améliorer notablement la précision de l'estimation de la valeur génétique du rendement en plante entière (Tableau 1). Cette augmentation de précision (Vincourt \& Gallais, 1983) est appréciée à travers l'augmentation du carré de la corrélation entre valeur génétique vraie et valeur génétique estimée $\left(r^{2}(\mathrm{G}, \widehat{\mathrm{G}})\right)$. Toutefois, les réponses corrélatives attendues de la hauteur totale et de la hauteur de l'épi (Tableau I) sont nettement plus importantes quand leurs valeurs phénotypiques sont prises en compte dans la sélection décrite ci-dessus. De plus, la réponse attendue de la hauteur de l'épi devient aussi importante que celle de la hauteur totale. Or une hauteur importante des plantes, et plus encore une position assez haute de l'épi, favorisent la sensibilité à la verse. Plutôt que de se priver du gain de précision sur le rendement en plante entière apporté par les mesures des hauteurs totale et de l'épi (la prise en compte simultanée de ces 2 caractères est souvent nécessaire), il est préférable d'imposer sur ceux-ci des contraintes de type inégalité. De telles contraintes réduisent inévitablement le progrès génétique attendu sur le rendement en plante entière. Son estimation est toutefois plus précise qu'en l'absence de mesure de caractères associés, et la sélection reste donc plus efficace.

Tableau I. Réponses attendues à la sélection dans une population de maïs fourrage précoce avec et sans prise en compte de caractères associés.

\begin{tabular}{|c|c|c|c|c|c|c|}
\hline $\begin{array}{l}\text { Caractères prédicteurs } \\
\text { retenus }\end{array}$ & $\begin{array}{l}r^{\prime}(G, \hat{G}) \\
r d t . f\end{array}$ & $\% m s$ & $\begin{array}{l}\text { Progr } \\
\text { rdt.f }\end{array}$ & $\begin{array}{l}\text { s génétiq } \\
\% m s\end{array}$ & $\begin{array}{l}\text { que at } \\
\text { ht }\end{array}$ & $\begin{array}{l}\text { ttendus } \\
\text { he }\end{array}$ \\
\hline rdt.f, \% ms & 0,57 & 0,78 & 0,64 & 0,00 & 3,0 & 2,7 \\
\hline $\begin{array}{l}\text { rdt.f, \% ms, ht, he, long, } \\
\text { d flo. }\end{array}$ & 0,70 & 0,84 & 0,84 & 0,00 & 4,8 & 4,7 \\
\hline
\end{tabular}

Pourcentage sélectionné $=15 \%$; rdt.f (tha) $:$ rendement en plante entière; $\% \mathrm{~ms}$ : teneur en matière sèche $(\%)$ du produit récolté; ht $(\mathrm{cm})$ : hauteur totale des plantes; he $(\mathrm{cm})$ : hauteur de l'épi; long (cm) : longueur de la feuille de l'épi; diflo : date de floraison femelle.

\section{UNE CONSÉQUENCE SUR LE CHOIX DES CRITERÈS DE SÉLECTION}

L'observation de caractères associés peut donc permettre d'augmenter la précision de l'estimation de la valeur génétique des caractères soumis à contrainte (appréciée par la valeur des coefficients $r^{2}(G, \hat{G})$ ). Toutefois, c'est l'ensemble des termes de la matrice $\Sigma_{\hat{\mathrm{G}} \hat{\mathrm{G}}}$ qui se trouve modifié par la prise en compte de caractères asso- 
ciés. Or, la valeur des progrès génétiques attendus pour un système de contraintes donné du type 5 ou 6 n'est pas seulement liée à la valeur des coefficients $r^{2}(G, \hat{G})$, mais dépend aussi de tous les termes de la matrice $\Sigma_{\hat{\mathrm{G}} \hat{\mathrm{G}}}$. Il est bien évident que l'on a intérêt à rechercher des valeurs élevées des coefficients $r^{2}(G, \widehat{G})$, de façon à obtenir une estimation suffisamment précise de la valeur génétique. Toutefois, les différents "jeux» de caractères observés conférant des valeurs élevées des coefficients $r^{2}(G, \hat{G})$ ne conduisent pas nécessairement aux mêmes espérances de progrès génétique pour un système de contraintes déterminé.

Ceci peut être vérifié sur des données expérimentales obtenues au GS Moulon pour le maïs fourrage. Une population de maiis précoce a été testée pour sa valeur en croisement en deux lieux (Arras et Le Moulon). Les essais ont été récoltés en plante entière. Des mesures de caractères de morphologie ont de plus été effectuées sur les essais implantés au Moulon. La sélection est effectuée sur un index des valeurs génétiques estimées du rendement en plante entière (rdt.f A) et de la teneur en matière sèche (\% ms A) des descendances en croisement à Arras. La prise en considération des données recueillies à la récolte des descendances en croisement au Moulon et des mesures de morphologie n'augmente pas la valeur du coefficient $r^{2}(G, G)$ des caractères retenus pour la sélection (Tableau II). Elle n'augmente pas non plus le progrès génétique attendu pour le rendement en plante entière des descendances en croisement à Arras (Tableau II) lorsqu'une perte de précocité (telle que $\Delta G \#-0,8$ points pour la teneur en matière sèche) est acceptée. Les caractères associés sont encore peu efficaces lorsqu'un maintien du niveau de précocité est souhaité ( $\Delta \mathrm{G}=0$ pour la teneur en matière sèche). Leur

Tableau II. Efficacité relative de caractères associés pour l'amélioration du rendement en plante entière, selon le niveau $(a, b, c)$ de progrès souhaité sur la précocité de récolte ( $\% \mathrm{~ms} A)$, dans une population de maïs fourrage précoce.

\begin{tabular}{|c|c|c|c|c|c|}
\hline \multirow[t]{2}{*}{$\begin{array}{l}\text { Caractères prédicteurs } \\
\text { observés }\end{array}$} & \multirow[t]{2}{*}{$r^{\prime}(G \hat{G})$} & \multirow[t]{2}{*}{$\begin{array}{l}\text { (1) rdt.fA } \\
\text { (2) } \% \mathrm{~ms} A\end{array}$} & \multicolumn{3}{|c|}{$\begin{array}{l}\text { Progrès génétique attendus } \\
\text { (1) rot. fA } \\
\text { (2) \% ms } A\end{array}$} \\
\hline & & & (a) & (b) & (c) \\
\hline \multirow{3}{*}{$\begin{array}{l}\operatorname{rdt} f \text { A, \% ms A } \\
\operatorname{rdt.f} A, \% \text { ms A, } \\
\operatorname{rdt} f \text { M, \% ms M, } \\
\text { ht M, he M }\end{array}$} & \multirow{3}{*}{ (1) } & 0,86 & (1) 2,00 & 1,71 & 1,00 \\
\hline & & 0,88 & $\begin{array}{r}(2-1,00 \\
2,04\end{array}$ & $\begin{array}{l}0,0 \\
1,80\end{array}$ & $\begin{array}{l}0,90 \\
1,20\end{array}$ \\
\hline & & 0,67 & $-0,80$ & 0,00 & 0,90 \\
\hline
\end{tabular}

Pourcentage sélectionné $=15 \%$; rdt.f (t/ha) : rendement en plante entière; $\% \mathrm{~ms}:$ teneur en matière sèche $(\%)$ du produit récolté; ht $(\mathrm{cm})$ : hauteur totale des plantes; he $(\mathrm{cm})$ : hauteur de l'épi supérieur; $M$ : caractères observés au Moulon : $A$ : caractères observés à Arras. prise en compte conduit par contre à une augmentation de près de $20 \%$ du progrès génétique attendu sur le rendement à Arras lorsqu'un gain de 0,9 points est souhaité pour la teneur en matière sèche. Le seul examen des coefficients $r^{2}(\mathrm{G}, \widehat{\mathrm{G}})$ aurait pourtant conduit à accorder peu d'intérêt aux caractères associés dans ce cas. La comparaison des ellipses de progrès génétique engendrées par les index $\mathrm{I}=\Sigma_{i} a_{i} \widehat{\mathrm{G}}_{i}$, avec et sans prise en compte des caractères associés, montre en effet une variation importante de leur "écartement» à l'intérieur du premier quadrant (Fig. 2).

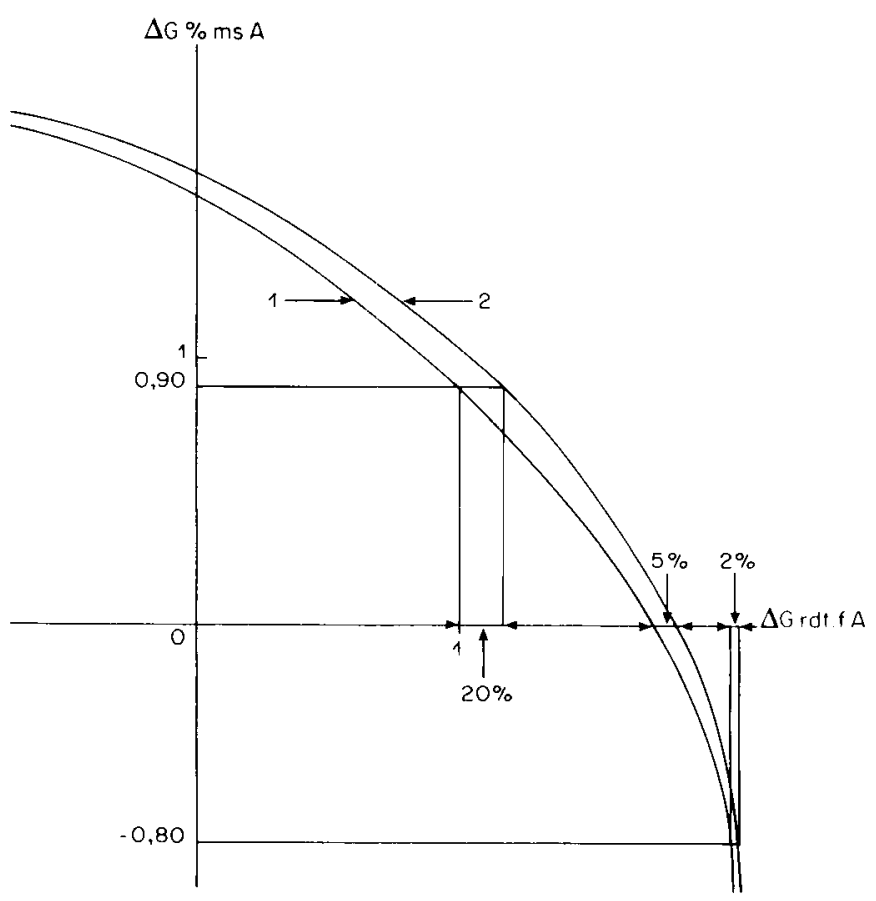

$\triangle G$ rdt.f A : progrès génétique attendu sur le rendement en fourrage à Arras ( $t / \mathrm{ha}$ $\triangle G \% \mathrm{~ms} \mathrm{~A}$ : progrès génétique attenou sur la teneur en matière sèche à Arras ( $p .100)$ Ellipse 1 : Caractères associés non pris en compte

Ellipse 2 : Caractères associés pris en compte (teis que décrits dans le tableau 2)

Fig. 2. Ellipses des espérances de progrès génétiques engendrées par les index $\mid=\Sigma_{i} a_{i} \widehat{G}_{i}$, avec et sans prise en compte de caractères associés, dans une population de maïs fourrage précoce.

\section{CONCLUSION}

La prise en compte d'un système de contraintes portant sur des gains désirés relatifs pour les différents caractères d'intérêt économique permet de déterminer les coefficients de l'index de façon pragmatique. Le choix d'un système de contraintes est toutefois une opération délicate. Le système de contraintes de stricte proportionnalité conduisant aux meilleures espérances de gains pour un objectif de sélection donné n'apparaît de façon évidente que dans des cas très simples. Dans tous les autres cas, il est nécessaire de rechercher le système de contraintes le moins 
restrictif possible par l'utilisation de contraintes d'inégalité; une détermination analytique des coefficients de l'index est alors nécessaire. L'emploi de contraintes d'inégalité est en particulier très opportun pour contrôler la réponse à la sélection de caractères associés. Dans le cadre de la recherche de critères de sélection, il apparaît nécessaire de vérifier l'intérêt d'un «jeu» de prédicateurs jusqu'à l'évaluation des progrès génétiques attendus pour le système de contraintes retenu; une estimation de la précision de ces valeurs attendues serait toutefois nécessaire de façon à pouvoir juger sans ambiguité de l'efficacité des prédicateurs.

\section{RÉFÉRENCES}

Gallais A. (1973) Sélection pour plusieurs caractères. Synthèse critique et généralisation. Ann. Amélior. Plant. 23, 183-208

Hazel L.N. (1943) The genetic basis for constructing selection indexes. Genetics 28, 476-490

Henderson C.R. (1963) Selection index and expected genetic advance. In : Statistical Genetics and Plant
Breeding (W.D. Hanson \& H.F. Robinson eds) Natl Acad. Sci. Nat. Res. Council, 982, 141-163

Henderson C.R. (1975) Best linear unbiased estimation and prediction under a selection model. Biometrics 21, 423-447

Itoh Y. \& Yamada Y. (1988) Selection indices for desired relative genetic gains with inequality constraints. Theor. Appl. Genet. 75, 731-735

Mallard J. (1972) La théorie et le calcul des index de sélection avec restrictions : synthèse critique. Biometrics 28, 713-735

Pesek J. \& Baker R.J. (1969) Desired improvement in relation to selection indices. Can. J. Plant. Sci. 49, 803-804

Rouvier R. (1969) Contribution à l'étude des index de sélection sur plusieurs caractères. Thèse Doctorat $3^{\mathrm{\theta}}$ cycle. Fac. Sci. Paris

Rouvier R. (1977) Mise au point sur le modèle classique d'estimation de la valeur génétique. Ann. Genet. Sel. Anim. 9, 17-26

Smith H.F. (1936) A discriminant function for plant selection. Ann. Eug. 7, 240-250

Vincourt P. \& Gallais A. (1983) Sur la recherche de critères de sélection : la régression géno-phénotypique. Agronomie 3, 827-830 